\title{
A giant thoracic duct cyst: An unusual cause of dysphagia
}

\author{
Atila Turkyilmaz, MD, and Atilla Eroglu, MD, Erzurum, Turkey
}

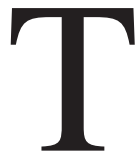

horacic duct cysts of the mediastinum, which may be of congenital or degenerative origin, are rare lesions. ${ }^{1-4}$ Although these cysts may occur anywhere along the course of the duct within the mediastinum, they are located in either the costovertebral sulcus or the visceral compartment of the mediastinum. We report a case of surgery on a giant thoracic duct cyst in an 82-year-old female patient. This rare cause of dysphagia is discussed alongside data from the literature.

\section{Clinical Summary}

The patient, an 82-year-old woman, was admitted with the chief symptom of dysphagia. A chest radiogram revealed an ovoid mass at the posterior mediastinum (Figure 1, a). An esophagogram showed deviation of the esophagus to the left and anterior. An esophagoscopic scan showed the middle esophagus to be narrowed by a compressing extrinsic lesion, but the esophageal mucosa appeared to be normal. A computed tomographic scan revealed a $9 \times 10$-cm, well-defined, homogeneous cystic lesion, with a lowdensity cyst bordering the esophagus, vertebral column, left lower pulmonary vein, and descending aorta. The esophagus was displaced by the cyst toward the anterior, and the descending aorta

From the Ataturk University, Medical Faculty, Department of Thoracic Surgery, Erzurum, Turkey.

Received for publication April 26, 2007; accepted for publication May 31, 2007.

Address for reprints: Atila Turkyilmaz, Department of Thoracic Surgery, Faculty of Medicine, Ataturk University, 25240 Erzurum, Turkey (E-mail: atilat@atauni.edu.tr, atilaeroglu@ hotmail.com).

J Thorac Cardiovasc Surg 2007;134:1082-3

$0022-5223 / \$ 32.00$

Copyright (C) 2007 by The American Association for Thoracic Surgery doi:10.1016/j.jtcvs.2007.05.043 was displaced by the cyst to the left (Figure $1, b)$. Magnetic resonance imaging of the thorax demonstrated well-defined lipid material in the hyperintense lesion in the posterior mediastinum (Figure 2,a). The left thoracotomy procedure was performed with the patient under general anesthesia. The cyst originated from the thoracic duct. A fine-needle aspiration revealed a milky fluid (Figure 2,b). These findings were consistent with the existence of a thoracic duct cyst. Because the cyst wall was contiguous to vital organs, mass ligation was performed on the thoracic duct to prevent postoperative chylothorax by incomplete resection. Postoperative recovery was uneventful, and the dysphagia disappeared.

\section{Discussion}

The thoracic duct originates in the abdomen at the level of the diaphragm and penetrates through the posterior and superior mediastinum, leaving the thorax through the thoracic aperture, usually posterior to the left innominate vein and left common carotid artery. ${ }^{5}$ Therefore, thoracic duct cysts are located in the costovertebral sulcus or the visceral compartment of the mediastinum. ${ }^{1,4,5}$

The cysts are symptomatic because of the pressure on adjacent structures. Symptoms such as coughing, dyspnea, and chest discomfort may be experienced. ${ }^{1,2,4}$ Symptoms of dysphagia are often associated with ingestion of fatty foods. ${ }^{2}$ Furthermore, acute respiratory insufficiency after ingestion of a fatty meal can be seen in some patients. ${ }^{2}$

Radiographically, these cysts appear as a round or oval, sharply circumscribed mass in the visceral compartment. ${ }^{1}$ Although computed tomography shows the cystic nature of the lesion, it does not differentiate it from any other mediastinal cystic lesion. ${ }^{2}$ Magnetic resonance imaging, especially $\mathrm{T} 2$-weighted images, better delin-

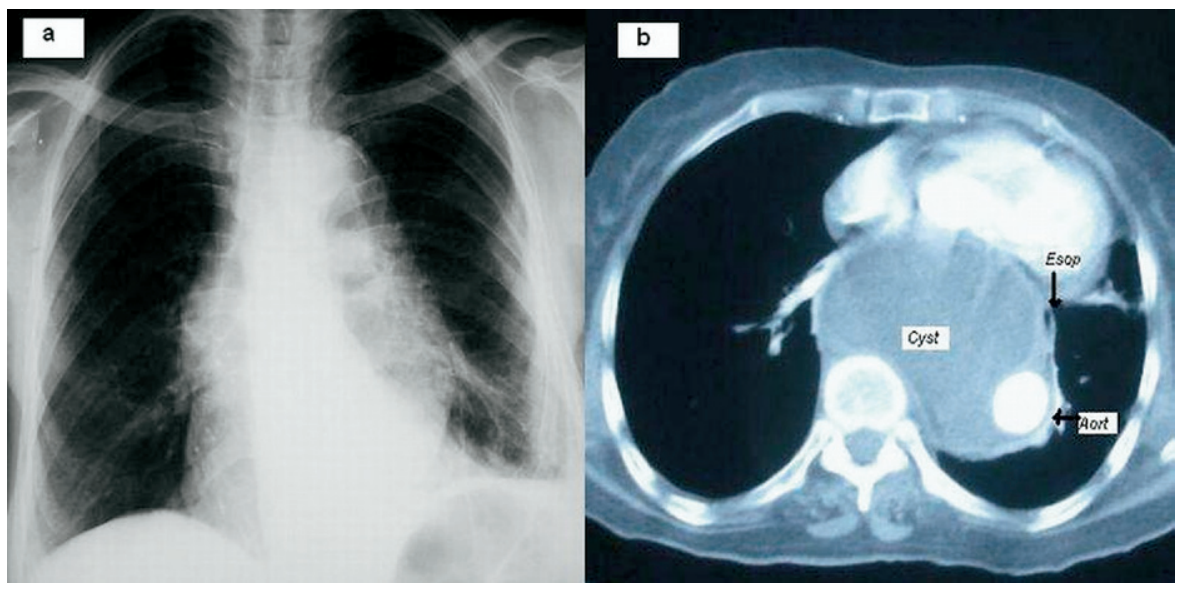

Figure 1. a, Chest radiogram showing a homogeneous left mediastinal mass. b, Axial computed tomographic scan showing a well-defined homogeneous, large cystic mass in the posterior mediastinum, displacing the esophagus, trachea, and descending aorta. 


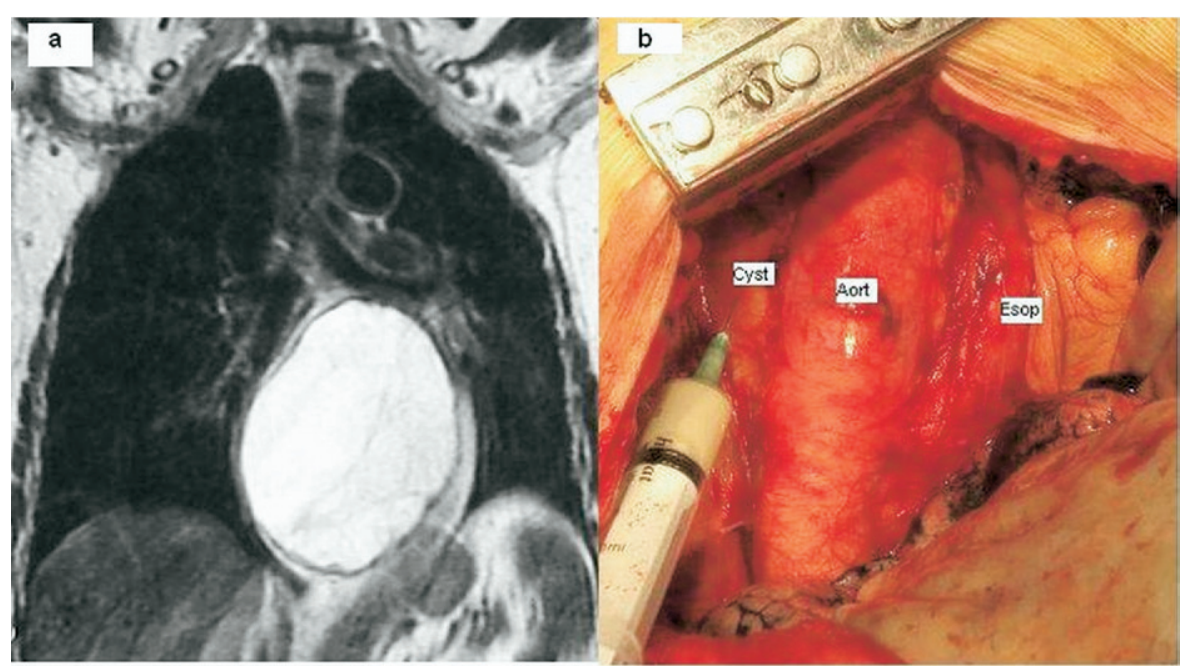

Figure 2. a, Coronal T2-weighted magnetic resonance image showing a highintensity thoracic duct cyst in an 82year-old female patient. $b$, Intraoperative fine-needle aspiration from the cyst.

eates the anatomic boundaries. The high signal intensity has been attributed to the high concentration of lipids and proteinaceous material in the cyst. ${ }^{1}$ Lymphangiograms may also assist with diagnosis. ${ }^{1,2}$ Fine-needle aspiration is a valuable diagnostic tool, yet its efficiency is debatable because of hypocellularity of the aspirate. ${ }^{4}$ Differential diagnosis includes pericardial or pleural mesothelial cysts, teratomatous cysts, bronchial or esophageal cysts, and neurenteric and lymphangiomatous cysts. ${ }^{2}$

Pathologically, the cysts are described as unilocular with an associated connection to the thoracic duct. Definitive diagnosis of mediastinal thoracic duct cysts is based on surgical findings and histopathologic analysis, finding a thick wall, including the endothelium and connective tissue, without elastic fiber. ${ }^{3}$

Surgical resection of the cyst is usually recommended. ${ }^{2,4}$ The surgical treatment should be performed to confirm the diagnosis arrived at by histologic analysis and to prevent potential complications such as spontaneous or traumatic rupture of the cyst. Surgical treatment consists of removal of the cyst and ligation of all lymphatics connected to it. ${ }^{4}$ When the localization of the cyst and invasion to vital structures prevent total excision, partial cystectomy is the treatment of choice. The most common complication seen after surgery is chylothorax requiring reoperation. To prevent this complication, the entire connection of the thoracic duct should be ligated. Video-assisted thoracic surgery may be an acceptable surgical procedure. ${ }^{2}$ Although surgery is the treatment of choice, ethanol sclerotherapy and an expectative approach in asymptomatic patients with confirmed diagnosis may represent an alternative therapy. ${ }^{1,3,5}$

Mediastinal thoracic duct cysts are rare, although they should be suspected in the differential diagnosis of patients with dysphagia. Surgical treatment of this cyst is necessary to confirm the diagnosis by histologic analysis and prevent potential complications such as spontaneous or traumatic rupture of the cyst.

\section{References}

1. Pramesh CS, Deshpande MS, Pantvaidya GH, Sharma S, Deshpande RK. Thoracic duct cyst of the mediastinum. Ann Thorac Cardiovasc Surg. 2003;9:264-5.

2. Karajiannis A, Krueger T, Stauffer E, Ris H. Large thoracic duct cyst - a case report and review of the literature. Eur J Cardiothorac Surg. 2000;17:754-6.

3. Chen F, Bando T, Hanaoka N, Terada Y, Ike O, Wada H, et al. Mediastinal thoracic duct cyst. Chest. 1999;115:584-5.

4. Moesgaard L, Baerentzen S, Mirz F. Cervical thoracic duct cyst: a differential diagnosis of left supraclavicular swelling. Eur Arch Otorhinolaryngol. 2007;264:797-9. Epub 2007 Feb 13.

5. Dool JJ, de Bree R, van den Berg R, Leemans CR. Thoracic duct cyst: sclerotherapy as alternative for surgical treatment. Head Neck. 2007; 29:292-5. 